\title{
An in vitro analysis of the output of salbutamol from different nebulizers
}

\author{
P.W. Barry, C. O'Callaghan
}

\begin{abstract}
An in vitro analysis of the output of salbutamol from different nebulizers. P.W. Barry, C. O'Callaghan. C) ERS Journals Ltd 1999.

ABSTRACT: The objective of this study was to determine the particle size and mass output of salbutamol from different nebulizers used under simulated breathing conditions.

Seven nebulizer/compressor combinations were assessed. Each nebulizer was charged with $5 \mathrm{mg}$ salbutamol solution and connected to a breathing simulator operating at tidal volumes of $150 \mathrm{~mL}$ and $600 \mathrm{~mL}$. Nebulizers were operated for $15 \mathrm{~min}$. Salbutamol collected on the filters was measured by liquid chromatography. Aerosol particle size was determined separately by laser diffraction.

The Pari LC Star nebulizer delivered the most salbutamol at both tidal volumes. The maximal output of the Medicaid Ventstream and Sidestream nebulizers was twothirds that of the LC Star, and they delivered less salbutamol than the LC Star or LC Plus nebulizers. The Intersurgical Cirrus nebulizer delivered the least salbutamol at both tidal volumes, although there was only a small difference between the Cirrus and Ventstream or Sidestream nebulizers at $150 \mathrm{~mL}$ tidal volume. The LC Plus nebulizer produced larger particles, mass median diameter $5.3 \mu \mathrm{m}$, compared with $3.6-4.0 \mu \mathrm{m}$ for the other nebulizers.

In conclusion, there were large differences in the delivery of salbutamol between the nebulizers studied, even between nebulizers of apparently the same class, and this should be borne in mind by regulatory authorities, clinicians and researchers. Eur Respir J 1999; 13: 1164-1169.
\end{abstract} \author{
Leicester, Leicester, UK. \\ Correspondence: P.W. Barry \\ Department of Child Health \\ University of Leicester \\ Clinical Sciences Building \\ Leicester Royal Infirmary \\ PO Box 65 \\ Leicester \\ LE2 7LX \\ UK \\ Fax: 441162523282
}

Dept of Child Health, University of

Keywords: Drug administration-

inhalation

nebulizers

salbutamol

Received: July 141998

Accepted after revision January 71999

This study was supported by Pari.
Nebulizers are widely used in the treatment of bronchoconstriction, particularly in acute severe asthma. No controlled study has been undertaken to determine the optimum dose of $\beta_{2}$-agonist to nebulize in acute severe asthma, but larger, more frequent doses are used in severe disease. As important as the nominal dose placed in the nebulizer is the huge variation in the amount of medication released from different nebulizers, suggesting that the choice of device may be as important as the choice of bronchodilator dose. Medical staff remain largely ignorant of the possible effects of nebulizer differences. This is particularly so in paediatrics, where results from studies on adults may be extrapolated, despite the obvious anatomical and breathing pattern differences between children and adults.

Nebulizers may be marketed without any information being available on specific drug outputs from the device. The situation has been complicated recently by the advent of newer nebulizer designs which enhance nebulizer output $[1,2]$, such as "open vent" nebulizers. These draw air into the nebulizer chamber, increasing the amount of drug aerosol produced for inhalation by the patient. "Breath enhanced, open vent" nebulizers have a valve system to control airflow through the nebulizer during tidal breathing. Increased nebulizer output occurs only during inspiration, and a smaller proportion of the output is therefore wasted. This type of nebulizer has been shown to double the delivery of salbutamol to the lung compared to a conventional nebulizer [3].
The output of "breath enhanced, open vent" nebulizers is affected by the patient's inspiratory flow [4], and they may have different output characteristics when used by children or adults with low inspiratory flows. Knowledge of the amount of drug that both an adult and a child are likely to inhale is important, not only to explain clinical effect when managing patients, but in order to interpret the increasing number of clinical trials where the actual dose of medication the subjects receive from drug delivery devices is ignored.

The aim of this study was to determine the particle size and mass output of the bronchodilator salbutamol from a number of different nebulizers when used under simulated breathing conditions. The conditions were chosen to represent the breathing pattern of a child and an adult, and the nebulizers to represent a range of different designs, including the newer "open vent" and "breath enhanced" systems.

\section{Methods}

\section{Nebulizers and medication}

Seven jet nebulizer/compressor combinations were assessed; the LC Plus nebulizer and Turboboy compressor (38G00, Pari GmbH, Starnberg, Germany), the LC Star nebulizer and Turboboy compressor (Pari), the Ventstream nebulizer and Portaneb compressor (Medicaid, Pagham, 
UK), the Sidestream nebulizer and Portaneb compressor (Medicaid) and the Cirrus nebulizer and Novair II compressor (Intersurgical Ltd, Wokingham, UK) were assessed with salbutamol nebulizer solution $(5 \mathrm{mg}$ in $2.5 \mathrm{~mL}$, Salamol Baker Norton, Harlow, UK). To simulate hospital use, the LC Star and Ventstream nebulizers were also assessed when driven by compressed air at $6 \mathrm{~L} \cdot \mathrm{min}^{-1}$. Six nebulizers of each type were used. Each was washed 10 times according to the manufacturers instructions prior to its first use.

\section{Particle size measurements}

Particle size distribution of the nebulizer output was measured by laser diffraction with a Malvern Mastersizer X (Malvern Ltd, Malvern, UK), which calculates particle size by measuring the diffraction angle of particles passed through a laser beam. Positioning of the nebulizer and presentation of the aerosol to the laser are important to obtain reproducible results [5]. In these experiments, nebulizers were charged with $2.5 \mathrm{~mL}(5 \mathrm{mg})$ of salbutamol nebulizer solution, placed $1-2 \mathrm{~cm}$ from the detector lens and $1 \mathrm{~cm}$ from the edge of the laser beam. Aerosol was drawn across the laser beam by suction from a vacuum pump placed opposite the nebulizer mouthpiece and $<3 \mathrm{~cm}$ from the laser beam.

A $100 \mathrm{~mm}$ detector lens was fitted to the Mastersizer, detecting particles from 0.5 to $180 \mu \mathrm{m}$ calculated diameter. The detector was programmed to make 2,000 measurements over $4 \mathrm{~s}$, starting after the nebulizer had been operating for $2 \mathrm{~min}$, and to only measure when between $5-25 \%$ of the laser beam was obscured by aerosol particles. Each nebulizer/compressor combination was tested four times.

\section{Breathing simulation}

Total drug output from the nebulizers was also measured using a breathing simulator, the Pari Sinus Breathing Simulator (Pari), which allows the simulated tidal volume, respiratory rate and inspiratory time to be independently adjusted. The experimental apparatus is illustrated in figure 1. Salbutamol nebulizer solution $(2.5 \mathrm{~mL} ; 5 \mathrm{mg})$ was added to the nebulizer, which was attached to the breathing simulator. Electrostatic filter pads were used to collect the aerosolized drug, housed in a plastic filter assembly (dead space $11 \mathrm{~mL}$ ). Nebulizers were connected to the filter assembly by the T-piece or mouthpiece supplied by the nebulizer manufacturer. Waste aerosol released during "expiration" was collected on the expiratory filter.

Two different breathing patterns were used, one to represent a child ("paediatric" breathing pattern), and one to represent an adult ("adult" breathing pattern). The tidal

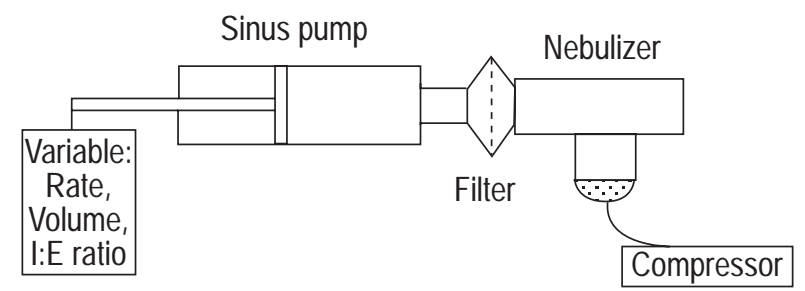

Fig. 1. - Schematic diagram of the breathing simulation apparatus. I:E ratio inhalation/expiration ratio. volume, respiratory rate and per cent inspiratory time for the paediatric breathing pattern were $150 \mathrm{~mL}, 20$ breaths. $\min ^{-1}$, and $40 \%$, and for the adult breathing pattern 600 $\mathrm{mL}, 12$ breaths $\cdot \mathrm{min}^{-1}$, and $40 \%$, respectively. These settings gave a minute volume, maximum inspiratory flow and mean inspiratory flow for the paediatric breathing pattern of $3 \mathrm{~L}, 11.8 \mathrm{~L} \cdot \mathrm{min}^{-1}$, and $7.5 \mathrm{~L} \cdot \mathrm{min}^{-1}$, and for the adult breathing pattern $7.2 \mathrm{~L}, 28.3 \mathrm{~L} \cdot \mathrm{min}^{-1}$, and $18 \mathrm{~L} \cdot \mathrm{min}^{-1}$, respectively.

Six nebulizers of each type were assessed at each breathing pattern for up to $15 \mathrm{~min}$. Nebulization was interrupted briefly after 1, 3, 5, 10 and 15 min to allow the inspiratory filter to be changed. In this way, the drug output at different times could be determined. The process of changing the filter took $<10 \mathrm{~s}$.

At the end of each experiment the filters were washed with an appropriate solvent. The amount of drug collected on the filters was determined using reversed phase highperformance liquid chromatography. A $10 \mathrm{~cm}$ Spherisorb ODS1 column (4.6 mm ID; Fisher Chemicals, Loughborough, UK) was used with methanol-0.1\% ammonium acetate as the mobile phase. The internal standard was benzyl biphenyl and ultraviolet detection was used at a wavelength of $276 \mathrm{~nm}$. The limit of detection of the assay was $<0.05 \mu \mathrm{g}$ salbutamol. $\mathrm{mL}^{-1}$. The system response was linear over a range of salbutamol concentrations from $0.3-$ $10 \mu \mathrm{g} \cdot \mathrm{mL}^{-1}$. The coefficient of variation of the assay was $6 \%$ (at a concentration of $0.5 \mu \mathrm{g} \cdot \mathrm{mL}^{-1}$ ).

In pilot studies, drug deposition on all parts of the experimental apparatus was measured, allowing the total recovery of drug to be estimated as a percentage of that added. A mean of $92.6 \%$ of the drug added was recovered (95\% confidence intervals (CI) 90.7-94.4\%). The three 15 min runs with the Sidestream nebulizer, where losses occurred from the mouthpiece, had recoveries of $<80 \%$. Apart from this, total drug recovery was not dependent upon the nebulizer type.

First minute and total output of salbutamol from the different nebulizers measured with the breathing simulator, and the percentage of the nebulizer output contained in particles $<5 \mu \mathrm{m}$ was compared using analysis of variance (ANOVA). Differences between the specific nebulizer types was inferred using the Tukey-Kramer method [6], which gives the 95\% CI for all pairwise differences between the mean recoveries. The time taken for the nebulizer to deliver $90 \%$ of the total output was calculated by interpolation from a graph of drug recovery plotted against time. Analysis was undertaken using Minitab statistical software (Clecom Ltd., Birmingham, UK).

\section{Results}

\section{Particle size}

The mass median diameter, the geometric SD, and the percentage of particles $<3$ and $<5 \mu \mathrm{m}$ diameter as measured by the Malvern Mastersizer are given in table 1 . The largest aerosol particles were produced by the LC Plus nebulizer, and the smallest by the LC Star driven by compressed air. The percentage of drug contained in particles $<5 \mu \mathrm{m}$ was similar for all nebulizers, approximately $70 \%$, except for the Cirrus and the LC Plus nebulizers, which delivered $57 \%$ and $49 \%$ of the drug, respectively, in particles $<5 \mu \mathrm{m}$ diameter under the test conditions. 
Table 1. - Aerosol particle size measured on the Malvern Mastersizer

\begin{tabular}{lcccc}
\hline Nebulizer/compressor & $\begin{array}{c}\text { MMD } \\
\mu \mathrm{m}\end{array}$ & GSD & $\begin{array}{c}\text { Particles }<5 \mu \mathrm{m} \\
\text { diameter } \%\end{array}$ & $\begin{array}{c}\text { Particles }<3 \mu \mathrm{m} \\
\text { diameter } \%\end{array}$ \\
\hline LC Plus/Turboboy & $5.1(4.9-5.3)$ & $2.0(1.9-2.1)$ & $49(46-52)$ & $22(19-24)$ \\
LC Star/Turboboy & $4.0(3.9-4.0)$ & $2.0(1.9-2.1)$ & $68(67-70)$ & $34(32-35)$ \\
LC Star/Air & $3.6(3.6-3.7)$ & $2.2(2.2-2.3)$ & $74(72-75)$ & $40(39-41)$ \\
SideStream/Portaneb & $3.9(3.9-4.1)$ & $1.9(1.8-2.1)$ & $69(68-70)$ & $33(31-35)$ \\
Ventstream/Portaneb & $3.8(3.5-4.1)$ & $2.1(2.0-2.2)$ & $71(64-77)$ & $37(33-41)$ \\
Ventstream/Air & $3.8(3.7-3.8)$ & $2.5(2.3-2.7)$ & $69(67-72)$ & $39(38-39)$ \\
Cirrus/Novair II & $4.4(4.1-4.6)$ & $3.2(3.1-3.2)$ & $58(54-61)$ & $35(33-37)$ \\
\hline
\end{tabular}

Data presented as means (95\% confidence intervals). MMD: mass median diameter; GSD: geometric standard deviation.

\section{Breathing simulation}

All nebulizers had a constant initial output of drug, which declined after a variable time (figs. 2 and 3 ). The maximum output rate (of drug) in the first minute, the total output over $15 \mathrm{~min}$, and the time taken for $90 \%$ of the total output to be released is reported in table 2 . There was a significant difference between the nebulizers in these parameters (ANOVA, $\mathrm{p}<0.0005$ ).

The LC Star nebulizer driven by the Turboboy compressor had a slightly smaller maximum output rate (mean (SD)) than the LC Plus (138.8 (23) $\mu \mathrm{g} \cdot \mathrm{min}^{-1}$ versus 176.9 (18) $\mu \mathrm{g} \cdot \mathrm{min}^{-1}$ with the "paediatric" breathing pattern, and $230.9(12.6) \mu \mathrm{g} \cdot \mathrm{min}^{-1}$ versus 251.5 (31.6) $\mu \mathrm{g} \cdot \mathrm{min}^{-1}$ at the "adult" breathing pattern), but continued nebulization for longer at both breathing patterns, and so delivered the most salbutamol over $15 \mathrm{~min}$. When driven with compressed air, the LC Star had a slightly higher maximal output rate, but delivered less salbutamol in total as the nebulization was completed sooner.

The Ventstream, Sidestream and Cirrus nebulizers delivered less salbutamol at both tidal volumes. The total output from these nebulizers was similar with the paediatric breathing pattern, but less for the Cirrus nebulizer with the adult breathing pattern. The time for these nebulizers to deliver $90 \%$ of the salbutamol was unaffected by the breathing pattern.

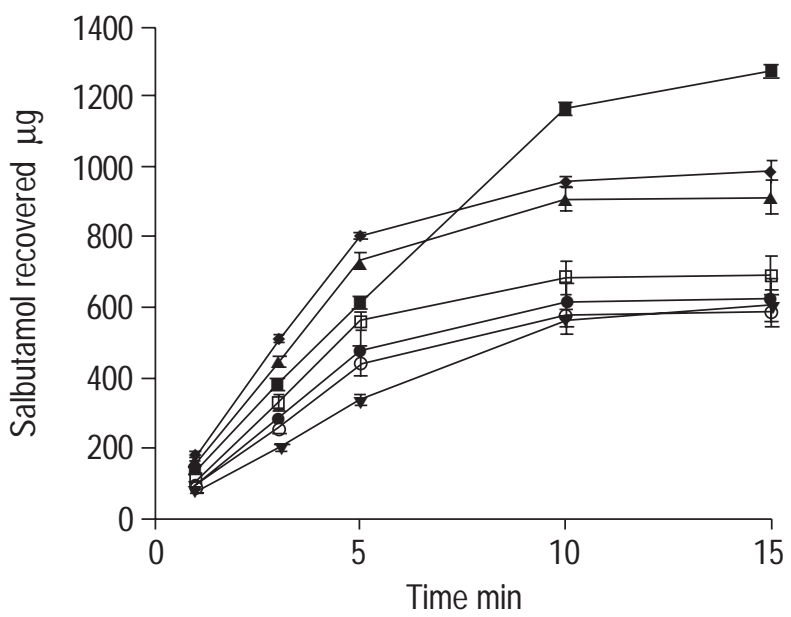

Fig. 2. - Amount of salbutamol collected on the filter of the breathing simulator over $15 \mathrm{~min}$, using the paediatric breathing pattern. Data presented as mean \pm SD. $\mathbf{\square}:$ LC Star+Turboboy; $\bullet$ : LC Plus+Turboboy; $\boldsymbol{\Delta}$ : LC Star+air $\left(6 \mathrm{~L} \cdot \mathrm{min}^{-1}\right)$; $\square$ : Sidestream+Portaneb; $\bigcirc$ : Ventstream+Portaneb; 0: Ventstream+air $\left(6 \mathrm{~L} \cdot \mathrm{min}^{-1}\right) ; \boldsymbol{\nabla}$ : Cirrus+Novair II.
To compare the differences between the different nebulizers, tables 3 and 4 give the $95 \%$ CI of the difference between the mean total output for the paediatric and adult breathing patterns respectively. This gives an indication of the precision of the estimate of the difference between the mean recoveries, taking into account the multiple comparisons made between nebulizers.

\section{Discussion}

Inhaled medications are increasingly used in the treatment of asthma and other respiratory diseases [7]. The inhalational route allows drug to be delivered directly to its site of action, and nebulizers are predominantly used by patients requiring high doses of medication and by those who are unable or unwilling to use other inhalational drug delivery devices. This in vitro study has detected large differences in the delivery of salbutamol from different nebulizers. Patients using one type of nebulizer may receive more than twice as much drug than another type. This may be crucial in acute severe asthma, where maximal drug delivery is required.

A number of other comparisons have demonstrated differences between nebulizers in vitro [8,9], although such comparisons often estimate the nebulizer output by weighing the nebulizer before and after a period of nebulization. This method allows the rapid assessment of

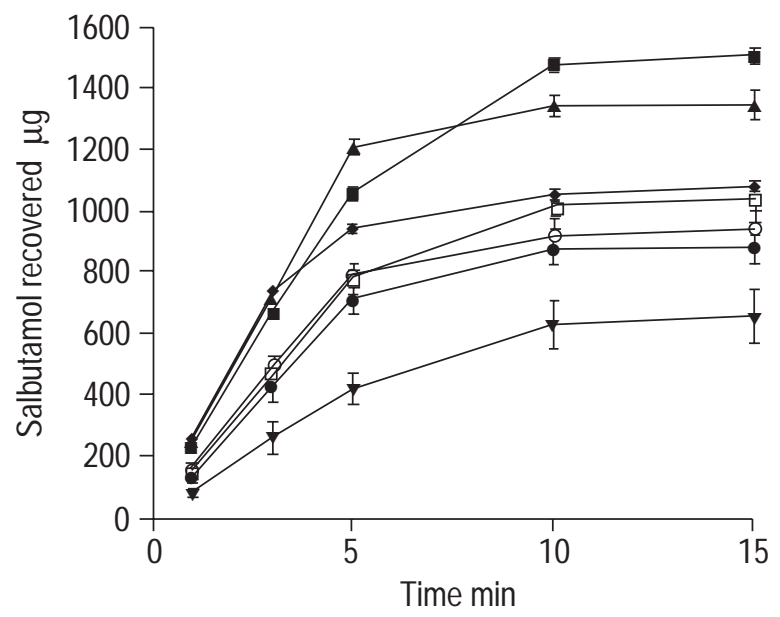

Fig. 3. - Amount of salbutamol collected on the filter of the breathing simulator over $15 \mathrm{~min}$, using the adult breathing pattern. Data presented as mean \pm SD. $\mathbf{\square}:$ LC Star+Turboboy; $\boldsymbol{\Lambda}$ : LC Star+air $\left(6 \mathrm{~L} \cdot \mathrm{min}^{-1}\right) ; \bullet:$ LC Plus+Turboboy; $\square$ : Sidestream+Portaneb; $\bigcirc$ : Ventstream+Portaneb; Ventstream+air $\left(6 \mathrm{~L} \cdot \mathrm{min}^{-1}\right) ; \boldsymbol{\nabla}$ : Cirrus+Novair II. 
Table 2. - Drug output

\begin{tabular}{|c|c|c|c|c|c|c|}
\hline \multirow[t]{2}{*}{ Nebulizer/compressor } & \multicolumn{2}{|c|}{ First minute output rate $\mu \mathrm{g} \cdot \mathrm{min}^{-1}$} & \multicolumn{2}{|c|}{ Total output over $15 \min \mu \mathrm{g}$} & \multicolumn{2}{|c|}{ Time to $90 \%$ output $\min$} \\
\hline & Paediatric & Adult & Paediatric & Adult & Paediatric & Adult \\
\hline LC Plus & $176.9(18.1)$ & $251.5(31.6)$ & $990.7(50.4)$ & $1085.6(53.4)$ & $7.8(0.3)$ & $6.1(1.3)$ \\
\hline LC Star/Turboboy & $138.8(23)$ & 230.9 (12.6) & $1273.1(36.5)$ & $1516.5(27.7)$ & $10.1(1.00)$ & $8.6(0.3)$ \\
\hline LC Star/Air & $148.2(21.5)$ & $248.7(50.2)$ & $917.6(12.7)$ & $1354.6(122.2)$ & $7.6(0.1)$ & $5.8(1.6)$ \\
\hline Sidestream & $90.7(20.2)$ & $157.0(22.6)$ & $595.6(54.7)$ & $1051.1(36.9)$ & $8.4(90.5)$ & $8.2(0.5)$ \\
\hline Ventstream/Portaneb & $102.9(8.4)$ & $156.7(18.2)$ & $693.2(56.3)$ & $950.0(59.8)$ & $7.3(1.1)$ & $7.3(0.8)$ \\
\hline Ventstream/Air & $90.4(15.6)$ & $145.4(21.4)$ & $627.4(58.8)$ & $893.8(57.0)$ & $7.8(0.9)$ & $7.3(0.9)$ \\
\hline Cirrus & $74.4(5.1)$ & $79.4(12.1)$ & $608.4(45.5)$ & $663.6(86.2)$ & $9.5(0.3)$ & $9.0(0.4)$ \\
\hline
\end{tabular}

Data presented as mean (SD).

a large number of nebulizers [10], but may be inaccurate [11]. Denyer and Dyche [12] used the more accurate tracer method [13] and breathing simulation to measure the output of the Ventstream nebulizer and a conventional nebulizer (the System 22 Acorn). The breathing simulator was set to a tidal volume of $630 \mathrm{~mL}$, frequency 20 breaths $\cdot \mathrm{min}^{-1}$, inspiratory fraction $33 \%$, fill volume $4 \mathrm{~mL}$ sodium fluoride solution and driving gas flow of $7 \mathrm{~L} \cdot \mathrm{min}^{-1}$. Output to the inspiratory filter from the Acorn nebulizer was $56 \%$ of that from the Ventstream, correlating well with a subsequent in vivo study [13] showing a two-fold increase in the delivery of salbutamol to the lungs of adult volunteers from the Ventstream compared to a conventional nebulizer. The present study suggests that this cannot be extrapolated to infant use, or to patients who have a low tidal volume. A recent study [14] compared the delivery of salbutamol onto filters from different nebulizers when used by a group of children aged 3-16 yrs. The study confirmed an increase in filter deposition with breath-enhanced nebulizers (Ventstream and LC Plus) than the conventional nebulizer (System 22 Acorn). The nebulizers were driven by compressed air at $6 \mathrm{~L} \cdot \mathrm{min}^{-1}$,

Table 3. - Differences between mean total drug recoveries for each pair of nebulizers using the paediatric breathing pattern

\begin{tabular}{lcccccc}
\hline & LC & $\begin{array}{c}\text { LC Star/ } \\
\text { Plus }\end{array}$ & $\begin{array}{c}\text { LC Star/ } \\
\text { Air }\end{array}$ & $\begin{array}{c}\text { Side- } \\
\text { stream }\end{array}$ & $\begin{array}{c}\text { Vent- } \\
\text { stream/ } \\
\text { PN }\end{array}$ & $\begin{array}{r}\text { Vent- } \\
\text { stream } \\
\text { Air }\end{array}$ \\
& & & & & & \\
LC Star/ & $-414-$ & & & & & \\
TB & -151 & & & & & \\
LC Star/ & $-59-$ & $224-$ & & & & \\
Air & 205 & 487 & & & & \\
Side- & $264-$ & $546-$ & $190-$ & & & \\
stream & 527 & 809 & 454 & & & \\
Vent- & $184-$ & $466-$ & $111-$ & $211-$ & & \\
stream/ & 412 & 694 & 339 & 16.4 & & \\
PN & & & & & & \\
Vent- & $249-$ & $532-$ & $176-$ & $-145-$ & $-27.3-$ & \\
stream/ & 477 & 760 & 404 & 82 & 159 & \\
Air & & & & & & \\
Cirrus & $268-$ & $551-$ & $195-$ & $-127-$ & $-8.4-$ & $-74-$ \\
& 496 & 779 & 423 & 101 & 178 & 112 \\
\hline
\end{tabular}

Data presented as $95 \%$ confidence intervals, expressed as the column mean minus the row mean, i.e. the difference between LC star/Turboboy nebulizer/compressor and the LC Plus nebulizer has a $95 \%$ probability of being between 151 and $414 \mu \mathrm{g}$. Where confidence intervals include zero, the difference was not statistically significant. Otherwise the values allow an estimate to be made of the clinical significance of the difference. TB: Turboboy; PN: Portaneb; Air: compressed air. apart from the LC Plus nebulizer which was evaluated with a low output compressor (Inhalierboy, driving gas flow $3.5 \mathrm{~L} \cdot \mathrm{min}^{-1}$ ), perhaps explaining why there was no difference between the two breath-enhanced nebulizers. The Turboboy compressor used in the present study had a driving gas flow of $4.3 \mathrm{~L} \cdot \mathrm{min}^{-1}$, and has been shown to have a greater output than the Inhalierboy when used with the LC Plus nebulizer [15].

Measurement of drug delivery using filter deposition is a useful noninvasive method of estimating drug delivery from different devices that overcomes the difficulties of measuring endpoints such as lung function in young children and the ethical problems of exposing subjects to radiation in radiolabelled deposition studies [16]. The breathing simulator allows parameters such as tidal volume and breathing frequency to be controlled, overcoming the large intrasubject variability seen when patients are used to "inhale" from the nebulizer [14, 17, 18], and which may make it difficult to determine whether there is a relationship between parameters such as age or size and drug delivery [14]. However, the dead space in the filter holder may lead to underestimations of the nebulizer output, especially at lower tidal volumes. This effect was minimized by having a filter with a low dead space (11 $\mathrm{mL}$ ), but may still be important for the $150 \mathrm{~mL}$ tidal volume. Also, the breathing simulator produces a sine wave flow pattern which may not reflect real breathing

Table 4. - Differences between mean total drug recoveries for each pair of nebuizers using the adult breathing pattern

\begin{tabular}{lcccccc}
\hline & LC & LC Star/ & LC Star/ & $\begin{array}{c}\text { Side- } \\
\text { stream }\end{array}$ & $\begin{array}{c}\text { Vent- } \\
\text { stream/ } \\
\text { PN }\end{array}$ & $\begin{array}{c}\text { Vent- } \\
\text { stream/ } \\
\text { Air }\end{array}$ \\
& & & & & PN & \\
& & & & & & \\
LC Star/ & $-614-$ & & & & & \\
TB & -248 & & & & & \\
LC Star/ & $-452-$ & $-21-$ & & & & \\
Air & -86 & 345 & & & & \\
Side- & $-149-$ & $283-$ & $121-$ & & & \\
stream & 217 & 648 & 486 & & & \\
Vent- & $-23-$ & $408-$ & $246-$ & $-57-$ & & \\
stream/ & 294 & 725 & 563 & 260 & & \\
PN & & & & & & \\
Vent- & $33-$ & $464-$ & $302-$ & $-1-$ & $-73-$ & \\
stream/ & 350 & 781 & 619 & 316 & 186 & \\
Air & & & & & & \\
Cirrus & $264-$ & $695-$ & $533-$ & $229-$ & $157-$ & $101-$ \\
& 581 & 1011 & 850 & 546 & 416 & 360 \\
\hline
\end{tabular}

For further explanation see footnote to table 3 . 
patterns [19], and filter deposition may not reflect clinical effect [20].

Drug collected on filters during the breathing simulation experiments represents all the medication "inhaled" by the patient, and is not the same as lung deposition. This will depend upon a number of factors, such as the mode of inhalation and the aerosol particle size [1]. The ideal particle size for lung deposition is not known, and may be dependent upon age [21] and decree of bronchoconstriction [22]. A particle diameter of $<5 \mu \mathrm{m}$ is thought to be appropriate for delineating between lung and extra-pulmonary deposition, but recent debate has suggested that a smaller particle size may be optimal [23]. The present study presents data on the percentage of particles $<5 \mu \mathrm{m}$ and $<3 \mu \mathrm{m}$ diameter.

A recent radiolabelled deposition study in adults using the LC Plus nebulizer found a mean lung deposition of $12.8 \%$ of the nominal dose [24]. In the present study with the same nebulizer, multiplying the percentage of the nominal dose deposited on the filter (during the adult pattern breathing simulation) by the fraction of drug in particles $<5 \mu \mathrm{m}$ (from laser diffraction) gives 10.8\%, a similar value. The same equation for the LC Star nebulizer gives a result of $20.6 \%$. Others have also suggested that lung deposition may be estimated from laboratory measurement of aerosol particle size and filter collection [25].

Despite evidence of correlation between in vitro and in vivo measurements of nebulizer output, there are clearly problems with the in vitro methods of measuring aerosol particle size and drug output described above. Inspiratory flow may alter the nebulizer output. KNOCH and WuNDERLICH [4] measured the output of drug solution from the LC Plus nebulizer at different inspiratory flows, and found that the output increased with inspiratory flow up to $20 \mathrm{~L} \cdot \mathrm{min}^{-1}$ but was unchanged above this. The droplet size decreased slightly between 0 and $20 \mathrm{~L} \cdot \mathrm{min}^{-1}$ inspiratory flow, decreasing further at higher flows. Calculations of lung deposition from in vitro data assume that the particle size output from the nebulizer throughout the simulated respiratory cycle is the same as that measured by the laser under a constant flow. The data of $\mathrm{KNOCH}$ and WUNDERLICH [4] suggests that this may not be so.

Laser diffraction measurements are dependent upon the exact alignment and positioning of the nebulizer and laser beam [5], as evaporation may occur from aqueous aerosols, distorting the measured particle size, and making the aerosol droplets appear smaller than other methods. In the present study, the positioning of the nebulizers was strictly controlled in an attempt to minimize the variability of this effect. New European standards for characterizing nebulizers will incorporate low flow cascade impactors rather than laser diffraction to overcome this potential problem (J. Dennis, University of Bradford, Bradford, UK, personal communication).

The results of this study only apply to the particular nebulizer brands tested, and may not apply to other nebulizers or the same nebulizers used with other medications. The differences highlighted should, however, be considered when testing other nebulizers. For instance, the output of salbutamol from the Sidestream nebulizer was flow dependent, increasing by over $75 \%$ at the adult breathing pattern. Had breathing simulation not been used, or the assessment not included the paediatric breathing pattern, erroneous conclusions might have been drawn. The current British standard for jet nebulizers (BS7711 part 3 [26]) does not include the effect of breathing pattern on nebulizer function, and this needs to be addressed in forthcoming standards for nebulizer assessment.

This study has demonstrated significant differences between nebulizers of the same type in their ability to nebulize salbutamol solution, and demonstrated the importance of considering the physiology of the patient when assessing drug delivery devices. It is hoped that this information will aid patients and physicians in the use of their inhalational drug delivery devices, and hence improve therapy.

\author{
Acknowledgements. The authors thank J. \\ Jackson for technical support.
}

\section{References}

1. $\mathrm{O}^{\prime}$ Callaghan $\mathrm{C}$, Barry PW. The science of nebulised drug delivery. Thorax 1997; 52 (Suppl. 2): S31-S44.

2. Nikander K. Drug Delivery Systems. J Aerosol Med 1994; 7: S19-S24.

3. Newnham DM, Lipworth BJ. Nebulizer performance, pharmacokinetics airways and systemic effects of salbutamol given via a novel nebulizer system (Ventstream). Thorax 1994; 49: 762-770.

4. Knoch M, Wunderlich E. In-vitro assessment of a new efficient nebulizer system for continuous operation. Conference proceedings, Respiratory Drug Delivery IV. Eaglewood, CO, Interpharm Press, 1994; pp. 265-271.

5. Bumiller MR. Low angle laser light scattering techniques for analysing pharmaceutical aerosols. International Society of Aerosols in Medicine. Conference proceedings, ISAM focus symposium, Puerto Rico, 1997.

6. Minitab Reference Manual. PA, Minitab Inc, 1995; p. 10.7 .

7. Muers MF. The rational use of nebulizers in clinical practice. Eur Respir Rev 1997; 44: 189-197.

8. Hurley PK, Smye SW. Performance assessment of a range of comercial jet nebulizers. Technol Health Care 1994; 1 : 209-214.

9. Loffert DT, lkle D, Nelson HS. A comparison of commercial jet nebulizers. Chest 1994; 106: 1788-1793.

10. Tandon R, McPeck M, Smaldone GC. Measuring nebulizer output. Aerosol production vs gravimetric analysis. Chest 1997; 111: 1200-1205.

11. O'Callaghan C, Clarke AR, Milner AD. Inaccurate calculation of drug output from nebulizers. Eur J Pediatr 1989; 148: 473-474.

12. Denyer J, Dyche T. Improving the efficiency of gas powered nebulizers. Eur Respir J 1993; 6 (Suppl. 17): $148 \mathrm{~S}$.

13. Dennis JH, Stenton SC, Beach JR, Avery AJ, Walters EH, Hendrick DJ. Jet and ultrasonic nebulizer output: use of a new method for direct measurement of aerosol output. Thorax 1990; 45: 728-732.

14. Devadason SG, Everard ML, Linto JM, Le Souef PN. Comparison of drug delivery from conventional versus 'Venturi' nebulizers. Eur Respir J 1997; 10: 2479-2483.

15. Nikander K, Wunderlich E. Output of budesonide suspension for nebulisation using different Pari jet nebulizer-compressor combinations. International Society of 
Aerosols in Medicine, conference proceedings, ISAM Focus Symposium, Tours, France, 1996.

16. Everard ML. Studies using radiolabelled aerosols in children. Thorax 1994; 49: 1259-1266.

17. Lodrup Carlsen KC, Nikander K, Carlsen KH. How much nebulised budesonide reaches infants and toddlers. Arch Dis Child 1992; 67: 1077-1079.

18. Thomas SHL, O'Doherty MJ, Page CJ, Nunan TO. Variability in the measurement of nebulised aerosol deposition in man. Clin Sci 1991; 81: 767-775.

19. Bisgaard H. Patient related factors in nebulised drug delivery to children. Eur Respir Rev 1997; 7: 376-377.

20. Vikre-Jorgensen J, Agertoft L, Pedersen S. Dose titration of nebulised budesonide in young children. Pediatr Pulmonol 1997; 23: 270-277.

21. Alderson PO, Secker-Walker RH, Strominger DB, Markham J, Hill RL. Pulmonary deposition of aerosols in children with cystic fibrosis. J Pediatr 1974; 84: 479-484.
22. Chung KF, Jevasingh K, Snashall PD. Influence of airway calibre on the intrapulmonary dose and distribution of inhaled aerosol in normal and asthmatic subjects. Eur Respir J 1988; 1: 890-895.

23. Newhouse MT. Is the current laboratory determination of respirable mass clinically relevant? International Society of Aerosols in Medicine. Conference proceedings, ISAM focus symposium, Puerto Rico, 1997.

24. Newman SP, Pitcairn GR, Hooper G, Knoch M. Efficient drug delivery to the lungs from continuously operated open vent nebulizer and low pressure compressor system. Eur Respir J 1994; 7: 1177-1181.

25. Smaldone GC. Drug delivery via aerosol systems: concept of aerosol inhaled. J Aerosol Med 1991; 4: 229235.

26. British Standards Institute. BS7711 part 3. Specification for gas powered nebulizers for the delivery of drugs. London, British Standards Institute 1994. 